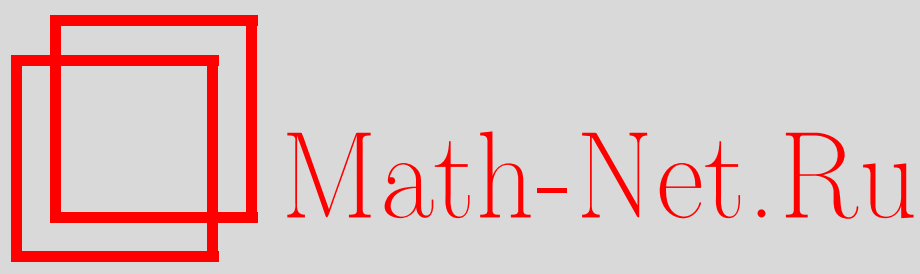

A. I. Karol', The singular values of compact pseudodifferential operators with spatially nonsmooth symbols, Sibirsk. Mat. Zh., 2020, Volume 61, Number 4, 849-866

DOI: https://doi.org/10.33048/smzh.2020.61.409

Use of the all-Russian mathematical portal Math-Net.Ru implies that you have read and agreed to these terms of use http://www . mathnet.ru/eng/agreement

Download details:

IP : 3.85 .73 .92

April 26, 2023, 05:50:54

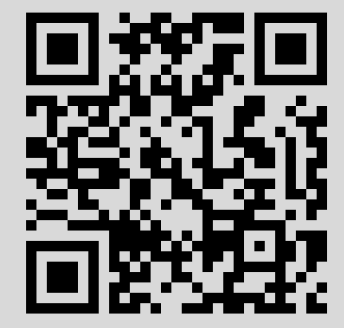


Сибирский математический журнал Июль-август, 2020. Том 61, № 4

УДК 517.9

\title{
СИНГУЛЯРНЫЕ ЧИСЛА КОМПАКТНЫХ ПСЕВДОДИФФЕРЕНЦИАЛЬНЫХ ОПЕРАТОРОВ С СИМВОЛОМ, НЕГЛАДКИМ ПО ПРОСТРАНСТВЕННЫМ ПЕРЕМЕННЫМ
}

\author{
А. И. Кароль
}

\begin{abstract}
Аннотация. Рассматриваются компактные псевдодифференциальные операторы с символами, гладкость которых по переменной $x$ нарушается на фиксированном множестве. Получены условия, когда для $s$-чисел таких операторов сохраняется вейлевская формула спектральной асимптотики. Результаты применяются к операторам, для которых порядок убывания символа по переменной $\xi$ является негладкой функцией от $x$.
\end{abstract}

DOI 10.33048/smzh.2020.61.409

Ключевые слова: псевдодифференциальный оператор, негладкий символ, сингулярные числа, вейлевская асимптотика.

\section{$\S 1$. Постановка задачи. Основные результаты}

Пусть $A: L_{2}\left(\mathbb{R}^{d}\right) \rightarrow L_{2}(\Omega)$ - компактный псевдодифференциальный оператор (ПДО),

$$
A u(x)=(2 \pi)^{-d} \int_{\mathbb{R}^{d}} \int_{\mathbb{R}^{d}} e^{i(x-y) \xi} a(x, \xi) u(y) d y d \xi, \quad u \in C_{0}^{\infty}\left(\mathbb{R}^{d}\right),
$$

область $\Omega \subset \mathbb{R}^{d}$ ограничена и имеет липшицеву границу. Будем считать, что символ $a$ определен для всех $x, \xi \in \mathbb{R}^{d}$ и $a(x, \xi) \equiv 0$ для всех достаточно больших $|x|$. Через $\left\{s_{k}(A)\right\}$ обозначим невозрастающую последовательность сингулярных чисел ( $s$-чисел) оператора $A$, занумерованную с учетом кратности, $s_{k}(A):=\sqrt{\lambda_{k}\left(A^{*} A\right)}, N(t, A)-$ функция распределения $s$-чисел,

$$
N(t, A):=\#\left\{k \mid s_{k}(A)>t^{-1}\right\} .
$$

В данной работе рассматриваются операторы с символами, гладкость которых по переменной $x$ нарушается на фиксированном множестве, а порядок убывания по переменной $\xi$ (локально - порядок оператора) может зависеть от точки $x$. Оценки и асимптотики спектра для ПДО с негладкими символами были ранее получены лишь для классических операторов с однородными по $\xi$ символами $[1,2]$. В этих работах использовался вариационный подход к вычислению спектральных асимптотик. Символ должен был принадлежать нужному

Работа поддержана грантом РФФИ № 18-01-00472.

(c) 2020 Кароль А. И. 
классу мультипликаторов ядер интегральных операторов. К операторам, у которых порядок убывания символа по $\xi$ зависит от точки $x$, эта техника неприменима. Спектральные асимптотики компактных ПДО с гладкими неклассическими символами в $\mathbb{R}^{d}$ рассматривались в $[3,4]$.

В $[5,6]$ рассматривались ПДО с вейлевскими символами, когда функция $a(x, \xi)$ в $(1)$ заменяется на $a((x+y) / 2, \xi)$. В [5] доказана справедливость вейлевской формулы спектральной асимптотики для неклассических ПДО с гладкими символами, в [6] - для операторов с вейлевскими символами, гладкость которых по переменной $x$ зависит от порядка оператора. Интерес к задачам определения спектральной асимптотики таких операторов возник в последнее время в связи с теорией случайных процессов. К этим задачам сводятся проблемы определения асимптотики вероятности малых уклонений в $L_{2}$ для гауссовых процессов с дробным переменным показателем Херста $[7,8]$. В этих задачах степенной порядок убывания символа при $|\xi| \rightarrow \infty$ является гёльдеровской функцией переменной $x$. Главный член спектральной асимптотики в этом случае определяется сколь угодно узкой окрестностью множества тех точек $x$, где порядок по абсолютной величине минимален. В [6] рассматриваются операторы с вейлевскими символами, в настоящей работе - с обычными, левыми, символами. Для операторов (1) удается существенно ослабить по сравнению с [6] требования гладкости символа.

Результаты работы анонсированы в [9].

Приведем необходимые обозначения. Через $C$ обозначаем различные константы, $\operatorname{vol}_{k}(D)-k$-мерный объем множества $D,\langle\xi\rangle:=\left(1+|\xi|^{2}\right)^{1 / 2}$. Для неотрицательной функции $f$ обозначаем через $\mu_{f}$ ее функцию распределения,

$$
\mu_{f}(s):=\operatorname{vol}_{d}\left\{x \in \mathbb{R}^{d} \mid 0<f(x)<s\right\} .
$$

Опишем нужный класс символов. Символ имеет особенности на множестве $\Gamma \subset \bar{\Omega}$. Фиксируем функцию $\mathrm{d}(x), x \in \mathbb{R}^{d}$, - регуляризованное расстояние до $\Gamma$ : $\mathrm{d} \asymp \operatorname{dist}(x, \Gamma)$, если $\operatorname{dist}(x, \Gamma)<1$, и $\mathrm{d}(x)=2$, если $\operatorname{dist}(x, \Gamma)>2$. Предположим, что $\mathrm{d}(x) \in C^{\infty}\left(\mathbb{R}^{d} \backslash \Gamma\right)$ и

$$
\left|\partial^{\alpha} \mathrm{d}(x)\right| \leq C(\alpha) \mathrm{d}^{1-|\alpha|}(x), \quad x \in \mathbb{R}^{d} \backslash \Gamma,
$$

а для некоторого $\varkappa>0$

$$
\mu_{\mathrm{d}}(s) \leq C s^{\varkappa}, \quad 0<s<1 .
$$

Условия (2), (3) заведомо выполнены, если Г - липшицева поверхность; в этом случае параметр $\varkappa$ является коразмерностью Г (см. [10]).

Через $\widetilde{S}_{\rho, \delta}^{-m}, m>0,0 \leq \delta<\rho \leq 1$, обозначим класс символов $a(x, \xi)$ таких, что для всех $\alpha, \beta$ при любом $\varepsilon>0$

$$
\left|\partial_{x}^{\alpha} \partial_{\xi}^{\beta} a(x, \xi)\right| \leq C(\alpha, \beta, \varepsilon)\langle\xi\rangle^{-m-|\beta| \rho+|\alpha| \delta+\varepsilon} \mathrm{d}^{-|\alpha|}(x), \quad x \in \mathbb{R}^{d} \backslash \Gamma, \xi \in \mathbb{R}^{d} .
$$

Отметим, что пересечение обычных классов Хёрмандера $S_{\rho^{\prime}, \delta^{\prime}}^{m^{\prime}}$ по всем $m^{\prime}>-m$, $\rho^{\prime}<\rho, \delta^{\prime}>\delta$, лежит в $\widetilde{S}_{\rho, \delta}^{-m}$. Оценки «с $\varepsilon \gg$ позволяют включать в рассмотрение символы, у которых убывание по переменной $\xi$ не строго степенное.

Напомним, что функция $f(t) \in C^{1}(1,+\infty), f(t) \neq 0$, называется медленно меняющейся (ММФ) на бесконечности, если $t f^{\prime}(t) / f(t) \rightarrow 0$ при $t \rightarrow+\infty$.

Сформулируем основные результаты работы. Пусть

$$
\operatorname{vol}_{2 d}\left\{x, \xi \in \Omega \times \mathbb{R}^{d}|| a(x, \xi) \mid>t^{-1}\right\}=t^{\frac{d}{m}} V(t, a),
$$


где $V(t, a)$ является медленно меняющейся функцией.

Следующее условие на символы класса $\widetilde{S}^{-m}$ будем накладывать лишь при $m \geq d / 2$, что соответствует операторам «почти Гильберта - Шмидта». Пусть для какого-нибудь $l \in(0,1)$ и любого $\varepsilon>0$

$$
|a(x, \xi)-a(y, \xi)| \leq C(\varepsilon)(1+|\xi|)^{-m+l+\varepsilon}|x-y|^{l}, \quad x, y, \xi \in \mathbb{R}^{d} .
$$

Теорема 1.1. Пусть для символа $a \in \widetilde{S}_{\rho, \delta}^{-m}$ выполнены условия (2)-(5). Если $m<d / 2$, то дополнительно потребуем, чтобы $\rho=1$, а если $m \geq d / 2$, то должны выполняться неравенства (6). Тогда для некоторого $\nu>0$ справедлива асимптотическая формула Вейля

$$
\begin{aligned}
N(t, A)=(2 \pi)^{-d} \operatorname{vol}_{2 d}\{x, \xi \in \Omega & \left.\times \mathbb{R}^{d}|| a(x, \xi) \mid>t^{-1}\right\}\left(1+O\left(t^{-\nu}\right)\right) \\
& =(2 \pi)^{-d} t^{\frac{d}{m}} V(t, a)\left(1+O\left(t^{-\nu}\right)\right), \quad t \rightarrow+\infty,
\end{aligned}
$$

$\nu=\nu(m, d, \delta, \varkappa, l)$.

Для операторов с вейлевскими символами при $m>d / 2$ в [6] по сравнению с настоящей работой накладывались гораздо более жесткие условия на гладкость символа вблизи множества Г.

В формуле (1) можно рассматривать ПДО $A$ на функциях $u$ из $C_{0}^{\infty}(\Omega)$. Cоответствующий оператор, действующий в $L_{2}(\Omega)$, обозначим через $A_{\Omega}$. Пусть в условиях теоремы 1 условие (5) заменяется условием поведения объема больших значений символа $\operatorname{Re} a(x, \xi)$ :

$$
\operatorname{vol}_{2 d}\left\{x, \xi \in \Omega \times \mathbb{R}^{d} \mid \operatorname{Re} a(x, \xi)>t^{-1}\right\}=t^{\frac{d}{m}} V_{R}(t, a),
$$

где $V_{R}$ - некоторая медленно меняющаяся функция. Для собственных чисел оператора $\operatorname{Re} A_{\Omega}:=\left(A_{\Omega}+A_{\Omega}^{*}\right) / 2$ справедлива

Теорема 1.2. Для функции распределения положительных собственных чисел оператора $\operatorname{Re} A_{\Omega}$ справедливо равенство

$$
\begin{aligned}
N_{+}\left(t, \operatorname{Re} A_{\Omega}\right):=\#\left\{k \mid \lambda_{k}\left(\operatorname{Re} A_{\Omega}\right)\right. & \left.>t^{-1}\right\} \\
& =(2 \pi)^{-d} t^{\frac{d}{m}} V_{R}(t, a)\left(1+O\left(t^{-\nu}\right)\right), \quad t \rightarrow \infty,
\end{aligned}
$$

где $\nu=\nu(m, d, \delta, \varkappa, l)>0$.

Опишем схему доказательства теорем 1.1 и 1.2. Представим оператор $A$ в виде суммы регулярной и сингулярной частей: $A=A_{0}+A_{1}$. Оператор $A_{0}$ является ПДО с гладким символом, к исследованию его спектра применим метод приближенного спектрального проектора. Для спектра регулярной части $A_{0}$ докажем асимптотическую формулу (7), здесь доказательство следует работе [5], где получена спектральная асимптотика компактных ПДО переменного порядка с гладкими символами. Далее, следуя [6], для спектра сингулярной части $A_{1}$ доказываются оценки, которые позволяют с помощью асимптотической теории возмущений сохранить асимптотику (7) для суммы $A_{0}+A_{1}$.

Спектральная асимптотика регулярной части в работе получена для символов из класса $a \in \widetilde{S}_{\rho, \delta}^{-m}, 0 \leq \delta<\rho \leq 1$. Условие $\rho=1$ возникает лишь при доказательстве оценок спектра сингулярной части, когда $m<d / 2$ (см. $\S 4$, теорема 4.1).

В теории случайных процессов возникает потребность в определении асимптотики $s$-чисел операторов с символом

$$
a(x, \xi)=(p(x, \xi))^{-(m+h(x))}, \quad m>d / 2, h \geq 0,
$$


где символ $p \in S_{\rho, \delta}^{1}$ эллиптичен. Функция $h$ в этих задачах принадлежит классу Гёльдера. Как указано выше, главный член спектральной асимптотики в этом случае определяется сколь угодно узкой окрестностью множества нулей функции $h$.

Пусть $D:=\{x \in \bar{\Omega} \mid h(x)=0\}, \mathrm{d}(x)$ - регуляризованное расстояние от точки $x$ до $D$. Будем считать, что вне $D$ для функции $\mathrm{d}(x)$ выполнены оценки (2). Пусть для некоторого $\nu_{0}>0$

$$
\operatorname{vol}_{d}\left\{\xi \in \mathbb{R}^{d}|| p(x, \xi) \mid<t\right\}=v(x) t^{d}\left(1+O\left(t^{-\nu_{0}}\right)\right), \quad t \rightarrow \infty .
$$

Теорема 1.3. Пусть $m>d / 2$ и $h \in C^{l}(\bar{\Omega})$ для какого-то $l \in(0,1)$. Предположим, что $h=h_{0}+h_{1}$, где $h_{0} \in C^{\infty}\left(\mathbb{R}^{d} \backslash D\right)$, причем

$$
\left|\partial^{\alpha} h_{0}(x)\right| \leq C(\alpha) h_{0}(x) \mathrm{d}^{-|\alpha|}(x), \quad x \in \mathbb{R}^{d} \backslash D,
$$

a $h_{1}=O\left(\left(h_{0}\right)^{1+\tau}\right)$ при $h_{0} \rightarrow 0$. Пусть для некоторой медленно меняющейся функции ч при $s \rightarrow+0$

$$
\int_{0<h(x)<s} v(x) d x=s^{\sigma} \varphi\left(s^{-1}\right), \quad \sigma>0,
$$

и при любом $\varepsilon>0$ справедлива оценка

$$
\mu_{h}(s) \leq C(\varepsilon) s^{\sigma-\varepsilon}, \quad 0<s<1
$$

Если $\operatorname{vol}_{d} D>0$, то при $\tau>\max \{0,(1-\sigma) / 2\}$ для функции распределения $s$-чисел оператора $A$ с символом (9) справедлива асимптотика

$$
N(t, A)=(2 \pi)^{-d} \int_{\Omega} t^{\frac{d}{m+h(x)}} v(x) d x+O\left(t^{d / m} \log ^{-r}(t)\right), \quad t \rightarrow \infty,
$$

для любого $r \in\left(0, \frac{2 \tau+\sigma-1}{2(d+m)} d\right)$.

Если $\operatorname{vol}_{d} D=0$, то при $\tau>\sigma(m / d-1 / 2)+1 / 2$

$$
N(t, A)=(2 \pi)^{-d} \int_{\Omega} t^{\frac{d}{m+h(x)}} v(x) d x\left(1+O\left(\log ^{-\nu}(t)\right)\right), \quad t \rightarrow \infty,
$$

где $\nu=\nu(m, d, \sigma, \tau, \varkappa)>0$.

Формула (13) является, конечно, реализацией формулы Вейля (5), (7) для оператора с символом (9). Вычисляя интегралы в (12), (13) методом Лапласа, получаем

Следствие. Если $\operatorname{vol}_{d} D>0$, то

$$
\begin{gathered}
N(t, A)=(2 \pi)^{-d} t^{\frac{d}{m}}\left(\alpha+\beta(\log t)^{-\sigma} \varphi(\log t)(1+o(1))\right)+O\left(t^{d / m} \log ^{-r} t\right) \quad t \rightarrow \infty \\
\alpha=\int_{D} v(x) d x, \quad \beta=\left(m^{2} / d\right)^{\sigma} \Gamma(\sigma+1)
\end{gathered}
$$

Г-Г-функция Эйлера.

Если $\operatorname{vol}_{d} D=0$, то

$$
\left.N(t, A)=(2 \pi)^{-d} \beta t^{\frac{d}{m}}(\log t)^{-\sigma} \varphi(\log t)(1+o(1))\right) .
$$


Отметим, что если $r \leq \sigma$, то в формуле (14) сохраняется лишь степенная асимптотика с логарифмической оценкой остатка.

Схема доказательства теоремы 1.3 повторяет в основном доказательство теорем $1.1,1.2$. Если в разложении функции $h$ слагаемое $h_{1}$ тождественно нулевое, то, поскольку символ $p \in S_{\rho, \delta}^{1}$ эллиптичен, символ $(9)$ попадает в класс $\widetilde{S}_{\rho, \delta}^{-m}$. Для такого оператора утверждение теоремы 1.3 со степенной оценкой остатка следует из теоремы 1.1. Тем самым в ПДО с символом (9) выделяется старшая часть. Остаток - оператор $A_{h_{1}}$ с символом $p(x, \xi)^{-\left(m+h_{0}(x)\right)}\left(p(x, \xi)^{-h_{1}(x)}-1\right)$, для его $s$-чисел доказывается оценка $N\left(t, A_{h_{1}}\right) \leq C t^{d / m} \log ^{-q} t$ с некоторым $q>\sigma$. Такие оценки через степени и логарифмы спектрального параметра получаются модификацией известных оценок $s$-чисел интегрального оператора через нормы ядра в соответствующих соболевских пространствах $[11,12]$.

Опишем структуру работы. В $\S 2$ приведены вспомогательные сведения об используемых пространствах Соболева и оценках $s$-чисел интегральных операторов, в 33 выделяется регулярная часть ПДО и приводятся результаты об асимптотике ее $s$-чисел. В $\S 4$ доказаны оценки $s$-чисел оставшейся сингулярной части, что дает доказательство теорем 1.1 и 1.2. В $\S 5$ рассматривается оператор переменного порядка с символом (9) и доказывается теорема 1.3.

\section{§ 2. Вспомогательные утверждения}

2.1. Сингулярные числа компактных операторов. Следующий результат об оценках $s$-чисел композиции операторов приведен в [13, теорема 2.2a].

Предложение 2.1. Пусть $T_{1}: H_{1} \rightarrow H_{2}, T_{2}: H_{2} \rightarrow H_{3}$ - компактные операторы такие, что для некоторых $p_{1}, p_{2}>0$ и медленно меняющихся функций $\Phi_{1}, \Phi_{2}$

$$
\limsup _{k \rightarrow \infty} s_{k}\left(T_{1}\right) k^{1 / p_{1}} \Phi_{1}(k) \leq M_{1}, \quad \limsup _{k \rightarrow \infty} s_{k}\left(T_{2}\right) k^{1 / p_{2}} \Phi_{2}(k) \leq M_{2} .
$$

Тогда для композиции $T_{2} T_{1}: H_{1} \rightarrow H_{3}$

$$
\limsup _{k \rightarrow \infty} s_{k}\left(T_{2} T_{1}\right) k^{1 / p} \Phi_{1}(k) \Phi_{2}(k) \leq \frac{p_{1}^{1 / p_{1}} p_{2}^{1 / p_{2}}}{\left(p_{1}+p_{2}\right)^{1 / p}} M_{1} M_{2}, \quad 1 / p=1 / p_{1}+1 / p_{2} .
$$

Ниже потребуются следующие результаты об оценках остатка в асимптотической теории возмущений.

Лемма 2.1. Пусть $A, B: H_{1} \rightarrow H_{2}-$ компактные операторы. Справедливы следующие утверждения.

1. Если для функций распределения $s$-чисел справедливы равенства $N(t, A)$ $=t^{p} V(t)$, где $V-\mathrm{MM} \Phi$, и $N(t, B)=O\left(t^{q}\right)$ при $t \rightarrow \infty$ для некоторого $q<p$, то для функции распределения $s$-чисел суммы операторов верно равенство

$N(t, A+B)=t^{p} V^{*}(t), \quad$ где $V^{*}(t)=V(t)+O\left(t^{-r}\right), r<(p-q) /(q+1)$.

2. Если $N(t, A)=t^{p} \log ^{-a} t V(\log t)$, где $V-\mathrm{MM \Phi ,} \mathrm{и} N(t, B)=O\left(t^{p} \log ^{-b} t\right)$ при $t \rightarrow \infty$ для некоторого $b>a$, то

$N(t, A+B)=t^{p} \log ^{-a} t V^{*}(\log t), \quad$ где $V^{*}(s)=V(s)+O\left(s^{-r}\right), r<(b-a) /(p+1)$.

3. Если $N(t, A)=t^{p}\left(\alpha+\beta \log ^{-a} t V(\log t)\right), a>0, V-M M \Phi, и N(t, B)=$ $O\left(t^{p} \log ^{-b} t\right)$ при $t \rightarrow \infty$ для некоторого $b>a$, то

$$
N(t, A+B)=t^{p}\left(\alpha+\beta \log ^{-a} t V(\log t)+O\left(\log ^{-r} t\right)\right), \quad \text { где } r<b /(p+1) .
$$


ДокАзАтельство. Утверждение п. 1 об оценках в степенной шкале имеется в [6, лемма 2.2]. Утверждения пп. 2 и 3 доказываются одинаково. Докажем утверждение п. 2.

Для функций распределения спектра известно неравенство

$$
N(t, A+B) \leq N\left(t_{1}, A\right)+N\left(t_{2}, B\right), \quad 1 / t=1 / t_{1}+1 / t_{2}
$$

$\left[12, \S 9.3\right.$, теорема 9]. Взяв $t_{2}=t \log ^{r} t$, получим, что $N\left(t_{2}, B\right)=O\left(t^{p} \log ^{-b+p r} t\right)$, а $N\left(t_{1}, A\right)=t^{p} \log ^{-a} t V(t)\left(1+O\left(\log ^{-r} t\right)\right)$. Таким образом,

$$
N(t, A+B) \leq t^{p} \log ^{-a} t V(t)\left(1+O\left(\log ^{-r} t\right)\right)+C t^{p} \log ^{-b+p r} t .
$$

Для $r<(b-a) /(p+1)$ отсюда следует, что $V^{*}(t) \leq V(t)+O\left(t^{-r}\right)$. Неравенство $V^{*}(t) \geq V(t)+O\left(t^{-r}\right)$, эквивалентное оценке

$$
N(t, A+B) \geq N(t, A)-C t^{p} \log ^{-b+p r} t,
$$

получается точно так же из равенства $A=(A+B)+(-B)$.

ЗАмечАниЕ 1. Результаты пп. 1 и 2 леммы справедливы и для функции распределения собственных чисел. Именно, если $H_{1}=H_{2}, A=A^{*}, B=B^{*}$ и для функции распределения положительных собственных чисел оператора $A$ справедливо $N_{+}(t, A)=t^{p} V_{+}(t)$, а для $s$-чисел оператора $B$ выполнена оценка $N(t, B)=O\left(t^{q}\right)$ при $t \rightarrow \infty$, то $N_{+}(t, A+B)=t^{p} V_{+}^{*}(t)$, где $V_{+}^{*}(t)=V_{+}(t)+O\left(t^{-r}\right)$, $r<(p-q) /(q+1)$.

Отметим, что, если в п. $3 b /(p+1) \leq a$, то для функции распределения суммы операторов сохраняется лишь степенная асимптотика с логарифмической оценкой остатка.

ЗАМЕчаниЕ 2. Оценка $s$-чисел

$$
\limsup _{k \rightarrow \infty} k^{p} \log ^{q} k s_{k} \leq C, \quad p>0,
$$

эквивалентна оценке функции распределения

$$
\limsup _{t \rightarrow \infty} N(t)^{p} t^{-1} \log ^{q} t \leq p^{q} C .
$$

2.2. Сведения по теории интерполяции. Приведем нужные факты из теории интерполяции. Пусть $\mathscr{H}_{1} \hookrightarrow \mathscr{H}_{0}-$ сепарабельные гильбертовы пространства с нормами $\|\cdot\|_{1},\|\cdot\|_{0}$ соответственно; $\mathscr{H}_{1}$ плотно и непрерывно вложено в $\mathscr{H}_{0}$. Будем говорить, что $\mathscr{H}_{1}, \mathscr{H}_{0}$ являются допустимой парой. Обозначим через $\mathscr{J}$ самосопряженный положительный оператор в $\mathscr{H}_{0}$ с областью определения $\mathscr{H}_{1}$ такой, что $\|\mathscr{J} u\|_{0}=\|u\|_{1}$ для всех $u$ из $\mathscr{H}_{1}$. Для параметров $\theta \in(0,1)$ и $q \in \mathbb{R}$ определим пространство $\mathscr{H}_{\theta, q}$ - область определения оператора $\mathscr{J}^{\theta} \log ^{q}(1+\mathscr{J})$ с нормой графика. Следующее предложение (см. [14, гл. 5.4.2]) означает, что функция $\mathbb{R}_{+} \ni t \rightarrow t^{\theta} \log ^{q}(2+t)$ является интерполяиионной функиией.

Предложение 2.2. Пусть $\mathscr{H}_{0}, \mathscr{H}_{1}$ и $\mathscr{V}_{0}, \mathscr{V}_{1}$ - допустимые пары и непрерывный оператор $T: \mathscr{H}_{1} \rightarrow \mathscr{V}_{1}$ продолжается до непрерывного оператора из $\mathscr{H}_{0}$ в $\mathscr{V}_{0}$. Тогда оператор $T$ непрерывно действует из $\mathscr{H}_{\theta, q}$ в $\mathscr{V}_{\theta, q}$, для соответствующих норм справедливо неравенство

$$
\|T\|_{\mathscr{H}_{\theta, q} \rightarrow \mathscr{V}_{\theta, q}} \leq C_{\theta, q} \max \left\{\|T\|_{\mathscr{H}_{0} \rightarrow \mathscr{V}_{0}},\|T\|_{\mathscr{H}_{1} \rightarrow \mathscr{V}_{1}}\right\} .
$$


2.3. Пространства Соболева. Через $H^{l, q}\left(\mathbb{R}^{d}\right), l>0,-\infty<q<\infty$, обозначим пространство Соболева функций с конечной нормой

$$
\|u\|_{H^{l, q\left(\mathbb{R}^{d}\right)}}^{2}:=\int_{\mathbb{R}^{d}}\langle\xi\rangle^{2 l}\left(1+\log ^{2}\langle\xi\rangle\right)^{q}|\hat{u}(\xi)|^{2} d \xi .
$$

Для $q=0$ получаем обычное пространство Соболева в $\mathbb{R}^{d}$, для этого случая сохраняем стандартное обозначение $H^{l}$. Пространства $H^{l, q}\left(\mathbb{R}^{d}\right)$ являются частным случаем пространств $B_{p, k}[15$, гл. 10.1] для $p=2$ и весовой функции $k(\xi)=\langle\xi\rangle^{l}(1+\log \langle\xi\rangle)^{q}$.

Очевидно, что пространства $H^{m}\left(\mathbb{R}^{d}\right)$ и $L_{2}\left(\mathbb{R}^{d}\right), m>0$, являются допустимой парой в смысле п. 2.2. При этом $\mathscr{J}=(1-\Delta)^{m / 2}$ и для $l=m \theta$ имеем $H^{l, q}\left(\mathbb{R}^{d}\right)=\mathscr{H}_{\theta, q}$, соответствующие нормы эквивалентны.

Для области $X \subset \mathbb{R}^{d}$ определим пространство $H^{l, q}(X)$ как пространство сужений функций из $H^{l, q}\left(\mathbb{R}^{d}\right)$ на $X$;

$$
\|u\|_{H^{l, q}(X)}:=\inf \left\{\|\tilde{u}\|_{H^{l, q}\left(\mathbb{R}^{d}\right)} \mid \tilde{u} \in H^{l, q}\left(\mathbb{R}^{d}\right), \tilde{u}(x)=u(x), x \in X\right\} .
$$

Если $X$ допускает непрерывный оператор продолжения $H^{m}(X) \rightarrow H^{m}\left(\mathbb{R}^{d}\right)$, $m>l$ (например, имеет липшицеву границу), то, конечно, пространства $H^{l, q}(X)$ интерполяционные в паре $H^{m}(X), L_{2}(X)$; норма (15) эквивалентна соответствующей интерполяционной норме для $l=m \theta$.

Для $l \in(0,1)$ удобно характеризовать принадлежность $H^{l, q}(X)$ в терминах разностных отношений. В случае $q=0$ утверждение следующей леммы хорошо известно.

Лемма 2.2. Пусть $l \in(0,1)$, а область $X$ имеет липшицеву границу (или $\left.X=\mathbb{R}^{d}\right)$. Для $u \in L_{2}(X)$ принадлежность пространству $H^{l, q}(X)$ эквивалентна конечности интеграла

$$
I_{\alpha}(u, X):=\int_{\substack{X \times X,|x-y|<\alpha}} \frac{|u(x)-u(y)|^{2}}{|x-y|^{d+2 l}}|\log | x-\left.y\right|^{2 q} d x d y, \quad 0<\alpha<1 .
$$

Таким образом, $I_{\alpha}(u, X)$ дает старшую часть эквивалентной нормы пространства $H^{l, q}(X)$. Для разных параметров $\alpha$ полученные нормы эквивалентны друг другу.

ДокАЗАТЕЛЬСтво. Разбиение единицы и локальные координаты стандартным образом сводят доказательство к случаям, когда область $X$ - это все пространство $\mathbb{R}^{d}$ или полупространство $\mathbb{R}_{+}^{d}$. Рассмотрим сначала случай $X=\mathbb{R}^{d}$.

Записав конечную разность через преобразование Фурье, получим равенство

$$
\int_{\mathbb{R}^{d}}|u(x+z)-u(x)|^{2} d x=(2 \pi)^{-d} \int_{\mathbb{R}^{d}}\left|e^{i z \xi}-1\right|^{2}|\hat{u}(\xi)|^{2} d \xi .
$$

Таким образом,

$$
I_{\alpha}\left(u, \mathbb{R}^{d}\right)=(2 \pi)^{-d} \int_{\mathbb{R}^{d}} \Phi(\xi)|\hat{u}(\xi)|^{2} d \xi, \quad \Phi(\xi):=\int_{|z|<\alpha} \frac{\left|e^{i z \xi}-1\right|^{2}}{|z|^{d+2 l}} \log ^{2 q}|z| d z .
$$

Функция $\Phi$ зависит лишь от $|\xi|$, после замены $z \rightarrow|\xi|^{-1} z$ имеем

$$
\Phi(\xi)=|\xi|^{2 l} \log ^{2 q}|\xi| \int_{|z|<\alpha|\xi|} \frac{\left|e^{i z \xi_{0}}-1\right|^{2}}{|z|^{d+2 l}}\left|\frac{\log |\xi|-\log |z|}{\log |\xi|}\right|^{2 q} d z .
$$


По теореме Лебега получаем

$$
\Phi(\xi)=\left.c_{0}|\xi|^{2 l}|\log | \xi\right|^{2 q}(1+o(1)),|\xi| \rightarrow \infty, \quad c_{0}=\int_{\mathbb{R}^{d}} \frac{\left|e^{i z \xi_{0}}-1\right|^{2}}{|z|^{d+2 l}} d z,\left|\xi_{0}\right|=1 .
$$

Подынтегральное выражение в (16) не превосходит

$$
C(\alpha) \frac{\left|e^{i z \xi_{0}}-1\right|^{2}}{|z|^{d+2 l}}(1+|\log | z||)^{2|q|},
$$

что дает суммируемую мажоранту для предельного перехода при $|\xi| \rightarrow \infty$. Таким образом, $\Phi(\xi) \asymp\langle\xi\rangle^{2 l}\left(1+\log ^{2}\langle\xi\rangle\right)^{q}$, и утверждение леммы для $X=\mathbb{R}^{d}$ доказано.

Пусть $X=\mathbb{R}_{+}^{d}$. Обозначим через $u_{e}$ четное продолжение функции $u$ через границу полупространства. Несложные выкладки приводят к неравенству $I_{\alpha}\left(u_{e}, \mathbb{R}^{d}\right) \leq 4 I_{\alpha}\left(u, \mathbb{R}_{+}^{d}\right)$, что вместе с утверждением леммы для $X=\mathbb{R}^{d}$ дает оценку

$$
\|u\|_{H^{l, q}\left(\mathbb{R}_{+}^{d}\right)}^{2} \leq c\left(I_{\alpha}\left(u, \mathbb{R}_{+}^{d}\right)+\|u\|_{L_{2}\left(\mathbb{R}_{+}^{d}\right)}^{2}\right) .
$$

Обратная оценка следует из очевидного неравенства $I_{\alpha}\left(u, \mathbb{R}_{+}^{d}\right) \leq I_{\alpha}\left(\tilde{u}, \mathbb{R}^{d}\right)$, где $\tilde{u} \in H^{l, q}\left(\mathbb{R}^{d}\right)$ - произвольное продолжение функции $u$ на $\mathbb{R}^{d}$.

Определим пространство $\widetilde{H}^{l, q}\left(Q_{s}\right)$ периодических функций в кубе $Q_{s}:=$ $(0,2 \pi s)^{d}$. Функция

$$
u(x)=\sum_{\mathbf{n} \in \mathbb{Z}^{d}} c_{\mathbf{n}} e^{i \mathbf{n} x / s}
$$

принадлежит этому пространству, если

$$
\|u\|_{\widetilde{H}^{l, q}\left(Q_{S}\right)}^{2}:=\sum_{\mathbf{n} \in \mathbb{Z}^{d}}\left(1+|\mathbf{n}|^{2}\right)^{l} \log ^{2 q}(2+|\mathbf{n}|)\left|c_{\mathbf{n}}\right|^{2}<\infty .
$$

Пространство $\widetilde{H}^{l, q}\left(Q_{s}\right)$ интерполяционное в паре $\widetilde{H}^{l_{0}}\left(Q_{s}\right)$ и $L_{2}\left(Q_{s}\right), l_{0}>$ $l>0$. Если для областей $X_{ \pm}$с липшицевой границей справедливы включения $X_{-} \subset Q_{s} \subset X_{+}$, то, конечно, справедливы вложения $H^{l, q}\left(X_{-}\right) \hookrightarrow \widetilde{H}^{l, q}\left(Q_{s}\right) \hookrightarrow$ $H^{l, q}\left(X_{+}\right)$.

Следующее утверждение является распространением на пространства $H^{l, q}$ известного результата об оценках $s$-чисел оператора вложения соболевских пространств $[12, \S 11.8]$. Пусть $X$ - ограниченная область в $\mathbb{R}^{d}$ с липшицевой границей, $J$ - оператор вложения $H^{l, q}(X) \hookrightarrow L_{2}(X)$.

Лемма 2.3. 1. Для $s$-чисел оператора $J$ справедлива оценка

$$
s_{k}(J) \leq C(X) k^{-\frac{l}{d}} \log ^{-q} k .
$$

2. Пусть

$$
(\mathscr{T} u)(x)=\int_{\mathbb{R}^{d}} T(x, y) u(y) d y
$$

- интегральный оператор, действующий из $L_{2}\left(\mathbb{R}^{d}\right)$ в $L_{2}(X)$, функция $x \rightarrow$ $T(x, y)$ для почти всех $y \in \mathbb{R}^{d}$ принадлежит пространству $H^{l, q}(X)$ и

$$
M^{2}:=\int_{\mathbb{R}^{d}}\|T(\cdot, y)\|_{H^{l, q}(X)}^{2} d y<\infty .
$$


Тогда для $s$-чисел оператора $\mathscr{T}$ справедлива оценка

$$
s_{k}(\mathscr{T}) \leq C_{X} M k^{-\left(\frac{1}{2}+\frac{l}{d}\right)} \log ^{-q} k .
$$

ДокаЗАТЕЛЬСтво. Фиксируем куб $Q_{s}$, содержащий область $X$, и обозначим через $\widetilde{J}$ оператор вложения $\widetilde{H}^{l, q}\left(Q_{s}\right) \hookrightarrow L_{2}\left(Q_{s}\right)$. Для $s$-чисел справедливо неравенство $s_{k}(J) \leq C s_{k}(\widetilde{J}), C-$ норма оператора продолжения $H^{l, q}(X) \rightarrow$ $\widetilde{H}^{l, q}\left(Q_{s}\right)$. Таким образом, утверждение п. 1 достаточно доказать для $s$-чисел оператора $\widetilde{J}$.

Разложение функции $u$ в ряд Фурье по системе экспонент в $Q_{s}$ можно записать в виде

здесь

$$
u=\sum_{\mathbf{n} \in \mathbb{Z}^{d}}\left(1+|\mathbf{n}|^{2}\right)^{-l / 2} \log ^{-q}(2+|\mathbf{n}|)\left(u, \phi_{\mathbf{n}}\right)_{\widetilde{H}^{l, q}\left(Q_{s}\right)} \psi_{\mathbf{n}},
$$

$$
\begin{gathered}
\psi_{\mathbf{n}}(x):=(2 \pi s)^{-d / 2} \exp (i \mathbf{n} x / s), \\
\phi_{\mathbf{n}}(x):=(2 \pi s)^{-d / 2}\left(1+|\mathbf{n}|^{2}\right)^{-l / 2} \log ^{-q}(2+|\mathbf{n}|) \exp (i \mathbf{n} x / s)
\end{gathered}
$$

- ортонормированные базисы в пространствах $L_{2}\left(Q_{s}\right)$ и $\widetilde{H}^{l, q}\left(Q_{s}\right)$ соответственно. Это разложение является рядом Шмидта оператора $\widetilde{J}$, а набор коэффициентов $\left(1+|\mathbf{n}|^{2}\right)^{-l / 2} \log ^{-q}(2+|\mathbf{n}|)$ - множеством его $s$-чисел. После упорядочивания этого набора получаем утверждение п. 1 леммы.

Условие п. 2 означает (см. $[12, \S 11.8]$ ), что оператор $\mathscr{T}$ является оператором Гильберта - Шмидта, действующим из $L_{2}\left(\mathbb{R}^{d}\right)$ в $H^{l, q}(X)$ с нормой, не превосходящей $M$. Для его $s$-чисел как оператора в этих пространствах имеется оценка $\lim \sup s_{k} k^{1 / 2} \leq \sqrt{2} M$. Таким образом,

$$
\mathscr{T}: L_{2}\left(\mathbb{R}^{d}\right) \stackrel{\mathscr{T}}{\longrightarrow} H^{l, q}(X) \stackrel{J}{\longrightarrow} L_{2}(X),
$$

и утверждение п. 2 следует из оценок п. 1 леммы и предложения 2.1.

\section{§3. Асимптотика спектра регулярной части}

Определим регулярную часть оператора (1), символ которого удовлетворяет условию (4). Зафиксируем срезки $\theta, \theta_{1} \in C^{\infty}(\mathbb{R})$,

$$
\theta(s)=0, s<1, \quad \text { и } \quad \theta(s)=1, s>2 ; \quad \theta_{1}(s):=1-\theta(s) .
$$

Построим функцию $\theta\left(\mathrm{d}(x)\langle\xi\rangle^{\delta^{\prime}}\right), \delta \geq \delta^{\prime}>0$. Из (2) следует, что $\theta\left(\mathrm{d}(x)\langle\xi\rangle^{\delta^{\prime}}\right) \in$ $S_{1, \delta^{\prime}}^{0}$, а из неравенств $(4)$ - что

$$
a_{0}(x, \xi):=a(x, \xi) \theta\left(\mathrm{d}(x)\langle\xi\rangle^{\delta^{\prime}}\right) \in S_{\rho, \delta_{0}}^{-m+\varepsilon}
$$

для любого $\varepsilon>0, \delta_{0}:=\delta+\delta^{\prime}$. Параметр $\delta^{\prime}>0$ выберем настолько малым, чтобы выполнялось неравенство $\delta_{0}<\rho$.

Назовем оператор $A_{0}$ с символом $a_{0}(x, \xi)$ регулярной частъю оператора $A$, оператор $A_{1}:=A-A_{0}-$ сингулярной частъю.

Оператор $A_{\Omega}: L_{2}(\Omega) \rightarrow L_{2}(\Omega)$ таким же образом представим как сумму регулярной и сингулярной частей, $A_{0, \Omega}+A_{1, \Omega}$.

Асимптотические свойства спектра регулярной части по существу получены в [6], где рассматривались операторы с вейлевскими символами. Обозначим через $W$ ПДО с вейлевским символом $w(x, \xi)$,

$$
(W u)(x):=(2 \pi)^{-d} \int_{\mathbb{R}^{d} \times \mathbb{R}^{d}} e^{i(x-y) \xi} w((x+y) / 2, \xi) u(y) d y d \xi, \quad u \in C_{0}^{\infty}(\Omega) .
$$

Напомним, что самосопряженность $W$ эквивалентна вещественности его вейлевского символа. 
Предложение 3.1 [6, теоремы 4.1, 4.2]. Пусть для объема больших значений вещественного символа $b \in \widetilde{S}_{\rho, \delta}^{-m}$ справедливо равенство

$$
\operatorname{vol}_{2 d}\left\{x, \xi \in \Omega \times \mathbb{R}^{d} \mid b(x, \xi)>t^{-1}\right\}=t^{\frac{d}{m}} V(t, b),
$$

где $V(t, b)$ является медленно меняющейся функцией, а вейлевский символ $w$ представлен в виде

$$
w(x, \xi)=b(x, \xi) \theta\left(\mathrm{d}(x)\langle\xi\rangle^{\delta^{\prime}}\right)+b_{1}(x, \xi), \quad b_{1} \in S_{\rho, \delta_{0}}^{-m_{1}}, m_{1}>m .
$$

Тогда для функции распределения положительных собственных чисел оператора $W$ в $L_{2}(\Omega)$ справедлива формула

$$
\begin{aligned}
N_{+}(t, W)=(2 \pi)^{-d} \operatorname{vol}_{2 d}\left\{x, \xi \in \Omega \times \mathbb{R}^{d} \mid b(x, \xi)>t^{-1}\right\}+O\left(t^{\frac{d}{m}-\nu_{0}}\right) & \\
& =(2 \pi)^{-d} t^{\frac{d}{m}} V(t, b)+O\left(t^{\frac{d}{m}-\nu_{0}}\right), \quad t \rightarrow \infty,
\end{aligned}
$$

$\nu_{0}=\nu_{0}\left(m, d, \rho, \delta_{0}, \delta^{\prime}, \varkappa\right)>0, \varkappa-$ параметр из условия (3).

Тем самым срезка $\theta$ и младшие члены символа сказываются лишь ухудшением оценки остатка асимптотики.

Из этого предложения сразу вытекает утверждение об асимптотике $N\left(t, A_{0}\right)$ и $N_{+}\left(t, \operatorname{Re} A_{0, \Omega}\right)$.

Следствие 3.1. В условиях теорем 1.1 и 1.2 соответственно

$$
\begin{gathered}
N\left(t, A_{0}\right)=(2 \pi)^{-d} t^{\frac{d}{m}} V(t, a)\left(1+O\left(t^{-\nu_{0}}\right)\right), \quad t \rightarrow \infty, \\
N_{+}\left(t, \operatorname{Re} A_{0, \Omega}\right)=(2 \pi)^{-d} t^{\frac{d}{m}} V_{+}(t, \operatorname{Re} a)\left(1+O\left(t^{-\nu_{0}}\right)\right), \quad t \rightarrow \infty,
\end{gathered}
$$

$\nu_{0}=\nu_{0}\left(m, d, \rho, \delta_{0}, \delta^{\prime}, \varkappa\right)>0$.

Доказательство. Очевидно, $N\left(t, A_{0}\right)=N_{+}\left(t^{2}, A_{0}^{*} A_{0}\right)=N_{+}\left(t^{2}, A_{0} A_{0}^{*}\right)$, а оператор $A_{0} A_{0}^{*}: L_{2}(\Omega) \rightarrow L_{2}(\Omega)$ является ПДО с амплитудой $a_{0}(x, \xi) \overline{a_{0}(y, \xi)}$ :

$$
\left(A_{0} A_{0}^{*} u\right)(x)=(2 \pi)^{-d} \int_{\mathbb{R}^{d} \times \Omega} e^{i(x-y) \xi} a_{0}(x, \xi) \overline{a_{0}(y, \xi)} u(y) d y d \xi .
$$

Этот оператор можно записать в виде (18) с помощью вейлевского символа $w(x, \xi)=\left|a_{0}(x, \xi)\right|^{2} \theta\left(\mathrm{d}(x)\langle\xi\rangle^{\delta^{\prime}}\right)^{2}+b_{1}(x, \xi), \quad b_{1} \in S_{\rho, \delta_{0}}^{-m_{1}}, 2 m<m_{1}<2 m+\rho-\delta_{0}$. Таким образом, формула (20) следует из равенства (12).

Точно так же исчисление ПДО позволяет представить оператор $\operatorname{Re} A_{\Omega}$ как оператор с вейлевским символом $w_{R}(x, \xi)=\operatorname{Re} a(x, \xi) \theta\left(\mathrm{d}(x)\langle\xi\rangle^{\prime}\right)+b_{1}(x, \xi), b_{1} \in$ $S_{\rho, \delta_{0}}^{-m_{1}}, m<m_{1}<m+\rho-\delta_{0}$. Это дает формулу $(21)$.

\section{$\S 4$. Оценки спектра сингулярной части}

Рассмотрим оператор $A_{1}:=A-A_{0}$. Для применения леммы 2.1 получим оценки функции $N\left(t, A_{1}\right)$. Оператор $A_{1}$ является интегральным оператором, действующим из $L_{2}\left(\mathbb{R}^{d}\right)$ в $L_{2}(\Omega)$, с ядром $T(x, y)=K(x, x-y)$,

$$
K(x, z):=(2 \pi)^{-d} \int_{\mathbb{R}^{d}} e^{i z \xi} a(x, \xi) \theta_{1}\left(\mathrm{~d}(x)\langle\xi\rangle^{\delta^{\prime}}\right) d \xi,
$$

где срезка $\theta_{1}$ введена в (17). Функция $K(x, z)$ определена для всех $x, z \in \mathbb{R}^{d}$, $K(x, z)=0$ при достаточно больших $|x|$, когда $\mathrm{d}(x) \geq 2$.

Доказательства в случаях $m<d / 2$ и $m \geq d / 2$ различны. 
Теорема 4.1. Пусть $a \in \widetilde{S}_{1, \delta}^{-m}, m<d / 2$. Тогда для достаточно больших $t$ справедлива оценка

$$
N\left(t, A_{1}\right) \leq C(p) t^{p}, \quad p>\max \left\{2,\left(d-\varkappa \delta^{\prime}\right) / m\right\} .
$$

ДокАЗАТЕЛЬСтво. Для операторов с вейлевскими символами такое же утверждение получено в [6, теорема 5.1]. Доказательство для операторов с левыми символами мало меняется.

Для параметра $\sigma>0$ определим ядра

$$
T_{\sigma}(x, y)=\left\{\begin{array}{ll}
T(x, y), & |x-y|<\sigma, \\
0, & |x-y|>\sigma,
\end{array} \quad T^{\sigma}(x, y)=T(x, y)-T_{\sigma}(x, y),\right.
$$

$\mathscr{T}^{\sigma}, \mathscr{T}_{\sigma}$ - соответствующие операторы, действующие из $L_{2}\left(\mathbb{R}^{d}\right)$ в $L_{2}(\Omega)$.

Для функции $K(x, z)$ справедливы оценки [6, лемма 5.1]

$$
|K(x, z)| \leq \begin{cases}\frac{C(l)}{|z|^{l}}, & l>d-m, \\ \frac{C(l, \varepsilon)}{|z|^{l} \mathrm{~d}(x)^{(d-m-l+\varepsilon) / \delta^{\prime}}}, & 0<l \leq d-m, \forall \varepsilon>0 .\end{cases}
$$

Тест Шура и неравенства (23) при $l=m+\varepsilon$ дают оценку нормы оператора $\mathscr{T}_{\sigma}$ :

$$
\left\|\mathscr{T}_{\sigma}\right\| \leq C(\varepsilon) \sigma^{m-\varepsilon} .
$$

Оценим с помощью неравенств (23) норму Гильберта - Шмидта оператора $\mathscr{T}^{\sigma}$. Для $d / 2<l<d-m$ (такой выбор возможен ввиду условия $m<d / 2$ ) имеем

$$
\begin{aligned}
\left\|\mathscr{T}^{\sigma}\right\|_{2}^{2}=\int_{\Omega \times \mathbb{R}^{d}}\left|T^{\sigma}(x, y)\right|^{2} d x d y & \leq \int_{\Omega} \int_{|z|>\sigma}|K(x, z)|^{2} d z d x \\
\leq C+\int_{\mathrm{d}(x)<2,|z|>\sigma} & \frac{c_{\varepsilon}}{|z|^{2 l}(\mathrm{~d}(x))^{2(d-m-l+\varepsilon) / \delta^{\prime}}} d x d z \\
& \leq C(\varepsilon) \sigma^{d-2 l} \int_{0}^{2} t^{-2(d-m-l+\varepsilon) / \delta^{\prime}} d \mu_{\mathrm{d}}(t) \stackrel{(*)}{\leq} C \sigma^{d-2 l} .
\end{aligned}
$$

Неравенство $(*)$ справедливо в силу условия (3), если $l>d-m-\varkappa \delta^{\prime} / 2$. Пользуясь известными неравенствами для $s$-чисел, получаем, что

$$
s_{k}\left(A_{1}\right) \leq s_{k}\left(\mathscr{T}^{\sigma}\right)+\left\|\mathscr{T}_{\sigma}\right\| \leq \sqrt{2} k^{-1 / 2}\left\|\mathscr{T}^{\sigma}\right\|_{2}+\left\|\mathscr{T}_{\sigma}\right\| \leq C_{\varepsilon} \sigma^{m-\varepsilon}\left(1+k^{-\frac{1}{2}} \sigma^{\frac{d}{2}-l-m+\varepsilon}\right) .
$$

Положим

$$
l=l\left(\varepsilon^{\prime}\right):=\max \left\{d / 2, d-m-\varkappa \delta^{\prime} / 2\right\}+\varepsilon^{\prime}<d-m, \quad \varepsilon^{\prime}>0,
$$

и выберем $\sigma$ так, чтобы выражение в скобках (24) было равно 2. Тогда $s_{k}\left(A_{1}\right) \leq$ $c_{l} k^{-1 / p\left(\varepsilon^{\prime}\right)}$, где параметр

$$
p\left(\varepsilon^{\prime}\right)=2+\frac{2 l\left(\varepsilon^{\prime}\right)-d}{m-\varepsilon}
$$

может быть любым большим $\max \left\{2,\left(d-\varkappa \delta^{\prime}\right) / m\right\}$. Полученная оценка $s$-чисел эквивалента оценке функции распределения (22).

Рассмотрим случай $m \geq d / 2$. 
Теорема 4.2. Пусть $a(x, \xi) \in \widetilde{S}_{\rho, \delta}^{-m}$ и для какого-то $l \in(0,1)$ выполнено условие (6). Тогда

$$
N\left(t, A_{1}\right) \leq C(p) t^{p}, \quad 1 / p<m / d+\delta^{\prime} \varkappa / 2 .
$$

ДокАЗАТЕЛЬСТво. Фиксируем функцию $b(y) \in C_{0}^{\infty}\left(\mathbb{R}^{d}\right), b(y)=1$ для $y$ в окрестности $\Omega$. Представим оператор $A_{1}$ в виде суммы: $A_{1}=\mathscr{T}_{b}+\mathscr{T}_{1-b}$,

$$
\mathscr{T}_{b} u(x)=(2 \pi)^{-d} \int_{\mathbb{R}^{d}} \int_{\mathbb{R}^{d}} e^{i(x-y) \xi} a(x, \xi) b(y) \theta_{1}\left(\mathrm{~d}(x)\langle\xi\rangle^{\delta^{\prime}}\right) u(y) d y d \xi,
$$

где срезка $\theta_{1}$ введена в $(17)$. Так как амплитуда $a(x, \xi)(1-b(y))$ обращается в нуль вблизи диагонали $\{x=y\}$, ядро оператора $\mathscr{T}_{1-b}$ является бесконечно гладкой функцией по $y$. Интегрированием по частям получаем, что она убывает быстрее любой степени $|y|$ на бесконечности. Отсюда следует, что

$$
s_{k}\left(\mathscr{T}_{1-b}\right) \leq C(N) k^{-N} \quad \forall N \in \mathbb{N}
$$

(см. [11, теорема 4.8]). Таким образом, основной вклад в оценку $s_{k}\left(A_{1}\right)$ вносит оператор $\mathscr{T}_{b}$,

$$
s_{k}\left(A_{1}\right) \leq C s_{k}\left(\mathscr{T}_{b}\right)+O\left(k^{-N}\right) \quad \forall N \in \mathbb{N} .
$$

Фиксируем параметр $m_{1}$

$$
m_{1}:=l+\left(d-\delta^{\prime} \varkappa\right) / 2+\varepsilon, \quad \varepsilon>0, m_{1}<m .
$$

Положим $m_{2}:=m-m_{1}$. Запишем оператор $\mathscr{T}_{b}$ в виде произведения $\mathscr{T}_{b}=R Q$, где $Q: L_{2}\left(\mathbb{R}^{d}\right) \rightarrow L_{2}\left(\mathbb{R}^{d}\right)$ и $R: L_{2}\left(\mathbb{R}^{d}\right) \rightarrow L_{2}(\Omega)$ :

$$
\begin{gathered}
(Q u)(\xi)=\int_{\mathbb{R}^{d}} e^{-i y \xi}\langle\xi\rangle^{-m_{2}} b(y) u(y) d y, \\
(R f)(x)=(2 \pi)^{-d} \int_{\mathbb{R}^{d}} e^{i x \xi} r(x, \xi) \theta_{1}\left(\mathrm{~d}(x)\langle\xi\rangle^{\delta^{\prime}}\right) f(\xi) d \xi, \\
r(x, \xi):=a(x, \xi)\langle\xi\rangle^{m_{2}} \in \widetilde{S}_{\rho, \delta}^{-m_{1}} .
\end{gathered}
$$

Лемма 4.1. Для $s$-чисел оператора $R$ справедливы оценки

$$
s_{k}(R) \leq C(\varepsilon) k^{-(1 / 2+l / d)+\varepsilon} \quad \forall \varepsilon>0 .
$$

ДокАЗАТЕЛЬСтво. В силу п. 2 леммы 2.3 для доказательства оценки (29) достаточно показать, что сходится интеграл

$$
\int_{\mathbb{R}^{d}}\|T(\cdot, \xi)\|_{H^{\ell}(\Omega)}^{2} d \xi, \quad \ell<l
$$

где $T(x, \xi)=e^{i x \xi} r(x, \xi) \theta_{1}\left(\mathrm{~d}(x)\langle\xi\rangle^{\delta^{\prime}}\right)$. По лемме 2.2 это равносильно сходимости интеграла

$$
\int_{\mathbb{R}^{d}} \int_{\Omega} \int_{\Omega} \frac{|T(x, \xi)-T(y, \xi)|^{2}}{|x-y|^{d+2 \ell}} d x d y d \xi
$$


Разобьем интеграл (30) на три части:

$$
\begin{gathered}
\int_{\mathbb{R}^{d}} \int_{\Omega} \int_{\Omega} \frac{\left|\left(e^{i(x-y) \xi}-1\right) r(x, \xi) \theta_{1}\left(\mathrm{~d}(x)\langle\xi\rangle^{\delta^{\prime}}\right)\right|^{2}}{|x-y|^{d+2 \ell}} d x d y d \xi, \\
\int_{\mathbb{R}^{d}} \int_{\Omega} \int_{\Omega} \frac{\left|(r(x, \xi)-r(y, \xi)) \theta_{1}\left(\mathrm{~d}(x)\langle\xi\rangle^{\delta^{\prime}}\right)\right|^{2}}{|x-y|^{d+2 \ell}} d x d y d \xi, \\
\int_{\mathbb{R}^{d}} \int_{\Omega} \int_{\Omega} \frac{\left|r(y, \xi)\left(\theta_{1}\left(\mathrm{~d}(x)\langle\xi\rangle^{\delta^{\prime}}\right)-\theta_{1}\left(\mathrm{~d}(y)\langle\xi\rangle^{\delta^{\prime}}\right)\right)\right|^{2}}{|x-y|^{d+2 \ell}} d x d y d \xi .
\end{gathered}
$$

Рассмотрим интеграл (31). Интеграл по переменной $y$ не превосходит $C(\ell, d)|\xi|^{2 \ell}$, где

$$
C(\ell, d)=\int_{\mathbb{R}^{d}}\left|e^{i z \xi_{0}}-1\right|^{2}|z|^{-(d+2 \ell)} d z, \quad\left|\xi_{0}\right|=1 .
$$

Учитывая также оценки функции $r \in \widetilde{S}_{\rho, \delta}^{-m_{1}}$, получаем, что интеграл $(31)$ не превосходит

$$
\begin{gathered}
C\left(\varepsilon^{\prime}\right) \int_{\mathbb{R}^{d}} \int_{\Omega}\langle\xi\rangle^{-2 m_{1}+2 \ell+2 \varepsilon^{\prime}} \theta_{1}^{2}\left(\mathrm{~d}(x)\langle\xi\rangle^{\delta^{\prime}}\right) d x d \xi \\
\leq C\left(\varepsilon^{\prime}\right) \int_{\mathbb{R}^{d}}\langle\xi\rangle^{-2 m_{1}+2 \ell+2 \varepsilon^{\prime}} \mu_{\mathrm{d}}\left(\langle\xi\rangle^{-\delta^{\prime}}\right) d \xi \\
\leq C\left(\varepsilon^{\prime}\right) \int_{\mathbb{R}^{d}}\langle\xi\rangle^{-2 m_{1}+2 \ell+2 \varepsilon^{\prime}-\delta^{\prime} \varkappa} d \xi \quad \forall \varepsilon^{\prime}>0 .
\end{gathered}
$$

Последний интеграл сходится, так как в силу (27) показатель степени $\langle\xi\rangle$ меньше $-d$.

Рассмотрим интеграл (32). Из условия (6) следует, что внутренний интеграл по переменной $y$ в $(32)$ не превосходит $\langle\xi\rangle^{-2 m_{1}+2 \ell+2 \varepsilon}$, и вновь приходим к оценке (34).

Оставшийся интеграл (33) оценивается через

$$
C\left(\varepsilon^{\prime}\right) \int_{\mathbb{R}^{d}}\langle\xi\rangle^{-2 m_{1}+2 \varepsilon^{\prime}}\left\|\theta_{1}\left(\mathrm{~d}(\cdot)\langle\xi\rangle^{\delta^{\prime}}\right)\right\|_{H^{\ell}\left(\mathbb{R}^{d}\right)}^{2} d \xi .
$$

Оценим норму функции $x \rightarrow \theta_{1}\left(\mathrm{~d}(x)\langle\xi\rangle^{\delta^{\prime}}\right)$ с помощью мультипликативного неравенства

$$
\left\|\theta_{1}\left(\mathrm{~d}(\cdot)\langle\xi\rangle^{\delta^{\prime}}\right)\right\|_{H^{\ell}\left(\mathbb{R}^{d}\right)} \leq C_{l}\left\|\nabla_{x} \theta_{1}\left(\mathrm{~d}(x)\langle\xi\rangle^{\delta^{\prime}}\right)\right\|_{L_{2}\left(\mathbb{R}^{d}\right)}^{\ell}\left\|\theta_{1}\left(\mathrm{~d}(x)\langle\xi\rangle^{\delta^{\prime}}\right)\right\|_{L_{2}\left(\mathbb{R}^{d}\right)}^{1-\ell} .
$$

Из (2) следует, что первая норма в правой части оценивается через $C\langle\xi\rangle^{2 \delta^{\prime}-\delta^{\prime} \varkappa}$, вторая - через $C\langle\xi\rangle^{-\delta^{\prime} \varkappa}$. Таким образом, интеграл (35) не превосходит интеграла

$$
C \int_{\mathbb{R}^{d}}\langle\xi\rangle^{-2 m_{1}+2 \varepsilon^{\prime}+2 \ell \delta^{\prime}-\delta^{\prime} \varkappa} d \xi
$$

который сходится ввиду (27). Лемма доказана. 
Закончим доказательство теоремы 4.2. Для $s$-чисел оператора $Q(28)$ известна оценка

$$
s_{k}(Q) \leq C(b) k^{-m_{2} / d}
$$

(см. $[1,11])$. Таким образом, в силу $(26)$ и предложения 2.1 для $s$-чисел оператора $A_{1}$ справедлива оценка

$$
s_{k}\left(A_{1}\right) \leq C s_{k}\left(\mathscr{T}_{b}\right)+O\left(k^{-n}\right) \leq C(\varepsilon) k^{-\left(1 / 2+l / d+m_{2} / d\right)+\varepsilon}=C(\varepsilon) k^{-\left(m / d+\delta^{\prime} \varkappa / 2\right)+\varepsilon} \forall \varepsilon .
$$

Это неравенство эквивалентно оценке функции распределения $s$-чисел (25).

ДокАЗАТЕЛЬСТво теОРЕм 1.1 и 1.2 сразу следует из результатов $\S 3,4$. Для регулярной части $A_{0}\left(A_{0, \Omega}\right)$ оператора $A\left(A_{\Omega}\right)$ справедливость асимптотических формул $(7),(8)$ является результатом следствия 3.1 ; оценки $s$-чисел сингулярной части, полученные в теоремах 4.1 и 4.2 , позволяют с помощью леммы 2.1 сохранить асимптотики (с ухудшением остатка) для оператора $A\left(A_{\Omega}\right)$.

\section{$\S 5$. Оператор переменного порядка}

Докажем теорему 1.3 для оператора $A$ с символом (9). Выделение главной части $h_{0}$ разложения функции $h, h=h_{0}+h_{1}$, в окрестности $\Gamma(\Gamma:=\partial D$, $D:=\{x \in \bar{\Omega} \mid h(x)=0\})$ задает главную часть оператора $A-$ оператор $A_{h_{0}} \mathrm{c}$ символом

$$
(p(x, \xi))^{-\left(m+h_{0}(x)\right)} \in \widetilde{S}_{\rho, \delta}^{-m} .
$$

По теореме 1.1 для функции распределения $s$-чисел этого оператора справедлива вейлевская асимптотическая формула (7), где объем больших значений символа вычисляется явно:

$$
N\left(t, A_{h_{0}}\right)=(2 \pi)^{-d} \int_{\Omega} t^{\frac{d}{m+h_{0}(x)}} v(x) d x\left(1+O\left(t^{-\nu}\right)\right), \quad t \rightarrow \infty, \nu>0 .
$$

Используя (10), получаем асимптотику интеграла Лапласа в (11):

$$
\begin{aligned}
N\left(t, A_{h_{0}}\right) & =(2 \pi)^{-d} t^{\frac{d}{m}}\left(\alpha+\beta(\log t)^{-\sigma} \varphi(\log t)(1+o(1))\right), \\
\alpha & =\int_{D} v(x) d x, \quad \beta=\left(m^{2} / d\right)^{\sigma} \Gamma(\sigma+1) .
\end{aligned}
$$

Оператор $A_{h_{1}}=A-A_{h_{0}}$ является ПДО с символом

$$
g(x, \xi):=p(x, \xi)^{-\left(m+h_{0}(x)\right)}\left(p(x, \xi)^{-h_{1}(x)}-1\right) .
$$

Получим оценки $s$-чисел оператора $A_{h_{1}}$.

Теорема 5.1. В условиях теоремы 1.3 для s-чисел оператора $A_{h_{1}}$ верна оценка

$$
s_{k}\left(A_{h_{1}}\right) \leq C_{q} k^{-m / d}(\log k)^{-q}, \quad q<\tau+(\sigma-1) / 2 .
$$

ДоКАЗАТЕЛЬСТво теоремы 5.1 проходит по той же схеме, что и доказательство теоремы 4.2. Фиксируем функцию $b \in C_{0}^{\infty}\left(\mathbb{R}^{d}\right), b(y)=1$ в окрестности $\Omega$, и умножим символ $(38)$ на функции $b(y)$ и $1-b(y)$. Получаем, что $A_{h_{1}}=\mathscr{S}_{b}+\mathscr{S}_{1-b}$, и $\mathscr{S}_{b}=G Q^{\prime}$, где $Q^{\prime}: L_{2}\left(\mathbb{R}^{d}\right) \rightarrow L_{2}\left(\mathbb{R}^{d}\right)$ и $G: L_{2}\left(\mathbb{R}^{d}\right) \rightarrow L_{2}(\Omega)$ :

$$
\left(Q^{\prime} u\right)(\xi)=\int_{\mathbb{R}^{d}} e^{-i y \xi}\langle\xi\rangle^{-m^{\prime}} b(y) u(y) d y,
$$




$$
G f(x)=(2 \pi)^{-d} \int_{\mathbb{R}^{d}} e^{i x \xi} g(x, \xi)\langle\xi\rangle^{m^{\prime}} f(\xi) d \xi .
$$

Фиксируем параметр $\ell, 0<\ell<\min \{l, m-d / 2\}$, и положим в формулах (40), (41)

$$
m^{\prime}=m-\ell-d / 2, \quad m^{\prime}>0 .
$$

У оператора $\mathscr{S}_{1-b} s$-числа, как и $s$-числа оператора $\mathscr{T}_{1-b}$ в доказательстве теоремы 4.2 , убывают сверхстепенным образом и не влияют на порядок убывания $s$-чисел оператора $A_{h_{1}}$. Для $s$-чисел оператора $Q^{\prime}$, как и для оператора $Q$ в доказательстве теоремы 4.2, справедливо неравенство

$$
s_{k}\left(Q^{\prime}\right) \leq C(b) k^{-m^{\prime} / d},
$$

так что утверждение теоремы вытекает с помощью предложения 2.1 из следующей леммы.

Лемма 5.2. Для s-чисел оператора $G$ верны неравенства

$$
s_{k}(G) \leq C k^{-(1 / 2+\ell / d)}(\log k)^{-q}, \quad q<\tau+(\sigma-1) / 2 .
$$

ДоКАЗАТЕЛЬСТво ЛЕМмЫ 5.2. В силу лемм 2.2 и 2.3 достаточно проверить конечность нормы ядра оператора $G$ в соответствующих пространствах Соболева:

$$
\begin{gathered}
\int_{\mathbb{R}^{d}}\|T(\cdot, \xi)\|_{H^{\ell, q}(\Omega)}^{2} d \xi \\
\quad \leq C \int_{\mathbb{R}^{d} \times \Omega \times \Omega,|x-y|<1 / 2} \frac{|T(x, \xi)-T(y, \xi)|^{2}}{|x-y|^{d+2 \ell}}|\log | x-y \|^{2 q} d x d y d \xi<\infty, \\
T(x, \xi)=e^{i x \xi} g(x, \xi)\langle\xi\rangle^{m^{\prime}} .
\end{gathered}
$$

Сходимость интеграла (43) вытекает из сходимости двух интегралов:

$$
\begin{gathered}
\int_{\mathbb{R}^{d} \times \Omega \times \Omega,|x-y|<1 / 2} \frac{\left|\left(e^{i x \xi}-e^{i y \xi}\right) g(x, \xi)\langle\xi\rangle^{m^{\prime}}\right|^{2}}{|x-y|^{d+2 \ell}}|\log | x-\left.y\right|^{2 q} d x d y d \xi<\infty, \\
\int_{\mathbb{R}^{d} \times \Omega \times \Omega,|x-y|<1 / 2} \frac{|g(x, \xi)-g(y, \xi)|^{2}\langle\xi\rangle^{2 m^{\prime}}}{|x-y|^{d+2 \ell}}|\log | x-\left.y\right|^{2 q} d x d y d \xi<\infty .
\end{gathered}
$$

Сначала рассмотрим интеграл (44). Как показано при доказательстве леммы 2.2, внутренний интеграл по переменной у равен $C(\ell, q)\langle\xi\rangle^{2 \ell} \log ^{2 q}\langle\xi\rangle(1+o(1))$ при $|\xi| \rightarrow \infty$. Подставив явное выражение (38) для символа $g$, приходим к оценке интеграла

$$
\int_{\mathbb{R}^{d} \times \Omega}\left|p(x, \xi)^{-\left(m+h_{0}(x)\right)}\left(p(x, \xi)^{-h_{1}(x)}-1\right)\right|^{2}\langle\xi\rangle^{2 \ell+2 m^{\prime}} \log ^{2 q}\langle\xi\rangle d x d \xi .
$$

Уменьшая параметр $\tau$ в оценке $h_{1}=O\left(h_{0}^{1+\tau}\right)$ при $h_{0} \rightarrow 0$, можно считать, что $h_{1}(x) \geq 0$. Фиксируем $\varepsilon>0$ и разделим здесь область интегрирования на две части:

$$
\mathscr{O}_{1}:=\left\{x, \xi \mid h_{0}^{1+\varepsilon}(x) \log \langle\xi\rangle<1\right\}, \quad \mathscr{O}_{2}:=\left\{x, \xi \mid h_{0}^{1+\varepsilon}(x) \log \langle\xi\rangle \geq 1\right\} .
$$


На части $\mathscr{O}_{1}$ имеем

$$
\left|p(x, \xi)^{-h_{1}(x)}-1\right| \leq h_{1}(x) \log |p(x, \xi)| \leq C h_{0}^{1+\tau}(x) \log \langle\xi\rangle,
$$

поскольку $|p(x, \xi)| \leq C\langle\xi\rangle$ (напомним, что $p \in S_{\rho, \delta}^{1}$ ). Используя эти неравенства, получаем с учетом (42), что интеграл (46) по $\mathscr{O}_{1}$ не превосходит

$$
\begin{gathered}
C \int_{\mathscr{O}_{1}}\langle\xi\rangle^{2 \ell+2 m^{\prime}-2 m-2 h_{0}(x)} \log ^{2+2 q}\langle\xi\rangle h_{0}^{2+2 \tau}(x) d x d \xi \leq C \int_{\mathbb{R}^{d}}\langle\xi\rangle^{-d} \log ^{2+2 q}\langle\xi\rangle I(\xi) d \xi, \\
I(\xi):=\int_{h_{0}^{1+\varepsilon}(x)<1 / \log \langle\xi\rangle} h_{0}^{2+2 \tau}(x) d x .
\end{gathered}
$$

Интеграл $I(\xi)$ выражается через функцию распределения $h_{0}$ и оценивается с использованием (11):

$$
\begin{gathered}
I(\xi)=\int_{0}^{\log -1 /(1+\varepsilon)\langle\xi\rangle} s^{2+2 \tau} d \mu_{h_{0}}(s) \leq C_{\varepsilon^{\prime}} \int_{0}^{\log ^{-1 /(1+\varepsilon)}\langle\xi\rangle} s^{2+2 \tau} s^{\sigma-\varepsilon^{\prime}-1} d s \\
\leq C_{\varepsilon^{\prime}}(\log \langle\xi\rangle)^{-\frac{2+2 \tau+\sigma-\varepsilon^{\prime}}{1+\varepsilon}} .
\end{gathered}
$$

Если $q<\tau+(\sigma-1) / 2$, то при достаточно малых $\varepsilon, \varepsilon^{\prime}>0$

$$
I(\xi) \leq C \log ^{-(2+2 q)-r}\langle\xi\rangle
$$

для какого-то $r>1$ и интеграл (47) по $\mathscr{O}_{1}$ конечен.

Рассмотрим интеграл (46) по оставшейся части $\mathscr{O}_{2}$. На этом множестве подынтегральное выражение не превосходит $C\langle\xi\rangle^{-\left(d+2 h_{0}(x)\right)} \log ^{2 q}\langle\xi\rangle$. Таким образом, достаточно проверить конечность интеграла

$$
\int_{\mathscr{O}_{2}}\langle\xi\rangle^{-\left(d+2 h_{0}(x)\right)} \log ^{2 q}\langle\xi\rangle d x d \xi .
$$

Выразим интеграл по переменной $x$ через функцию распределения $h_{0}$ и сделаем замену $s \log \langle\xi\rangle=w$ :

$$
\begin{aligned}
\int_{h_{0}^{1+\varepsilon}(x)>1 / \log \langle\xi\rangle}\langle\xi\rangle^{-2 h_{0}(x)} d x & =\int_{s^{1+\varepsilon}>1 / \log \langle\xi\rangle} e^{-2 s \log \langle\xi\rangle} d \mu_{h_{0}}(s) \\
\leq C\left(\varepsilon^{\prime}\right) & \int_{s^{1+\varepsilon}>1 / \log \langle\xi\rangle} e^{-2 s \log \langle\xi\rangle} s^{\sigma-\varepsilon^{\prime}-1} d s \\
\leq C\left(\varepsilon^{\prime}\right) \log \sigma-\varepsilon^{\prime}\langle\xi\rangle & \int_{w>(\log \langle\xi\rangle) \varepsilon /(1+\varepsilon)} e^{-2 w} w^{\sigma-\varepsilon^{\prime}-1} d w .
\end{aligned}
$$

Последний интеграл есть $\left(O(\log \langle\xi\rangle)^{-\infty}\right)$, и интеграл $(48)$ конечен для любого $q$. Таким образом, интеграл (44) сходится.

Рассмотрим интеграл (45). Положим

$$
b(x, \xi, h):=(p(x, \xi))^{-(m+h)} .
$$


В интеграле (45) имеем $g(x, \xi)=b(x, \xi, h(x))-b\left(x, \xi, h_{0}(x)\right)$ и

$$
|g(x, \xi)-g(y, \xi)| \leq|b(x, \xi, h(x))-b(y, \xi, h(y))|+\left|b\left(x, \xi, h_{0}(x)\right)-b\left(y, \xi, h_{0}(y)\right)\right| .
$$

Докажем сходимость интеграла с функцией $h(x)$ (сходимость другого интеграла проверяется так же):

$$
\int_{\mathbb{R}^{d} \times \Omega \times \Omega,|x-y|<1 / 2} \frac{|b(x, \xi, h(x))-b(y, \xi, h(y))|^{2}\langle\xi\rangle^{2 m^{\prime}}}{|x-y|^{d+2 \ell}}|\log | x-\left.y\right|^{2 q} d x d y d \xi<\infty .
$$

Прибавляя и вычитая в числителе дроби функцию $b(x, \xi, h(y))$, приходим к проверке конечности двух интегралов

$$
\begin{aligned}
& \int_{\substack{\mathbb{R}^{d} \times \Omega \times \Omega,|x-y|<1 / 2}} \frac{|b(x, \xi, h(x))-b(x, \xi, h(y))|^{2}\langle\xi\rangle^{2 m^{\prime}}}{|x-y|^{d+2 \ell}}|\log | x-\left.y\right|^{2 q} d x d y d \xi<\infty, \\
& \quad \int \frac{|b(x, \xi, h(y))-b(y, \xi, h(y))|^{2}\langle\xi\rangle^{2 m^{\prime}}}{|x-y|^{d+2 \ell}}|\log | x-\left.y\right|^{2 q} d x d y d \xi<\infty . \\
& \qquad \underset{\substack{\mathbb{R}^{d} \times \Omega \times \Omega,|x-y|<1 / 2}}{\mid x}
\end{aligned}
$$

Рассмотрим сначала интеграл (49). Оценим числитель дроби в подынтегральном выражении:

$(b(x, \xi, h(y))-b(y, \xi, h(y)))\langle\xi\rangle^{m^{\prime}}=p(x, \xi)^{-m}\langle\xi\rangle^{m^{\prime}}\left(e^{-h(x) \log p(x, \xi)}-e^{-h(y) \log p(x, \xi)}\right)$.

Можно считать, что $|p(x, \xi)|>1$, так что $\operatorname{Re} \log p(x, \xi)>0$. Выражение в больших скобках оценивается с помощью неравенства $\left|e^{-z_{1}}-e^{-z_{2}}\right| \leq\left|z_{1}-z_{2}\right|$, где $\operatorname{Re} z_{1}, z_{2}>0$. С учетом соотношения показателей (42) выражение (51) по модулю не превосходит

$$
C\langle\xi\rangle^{-(\ell+d / 2)}|h(x)-h(y)| \log |p(x, \xi)| \leq C(\varepsilon)\langle\xi\rangle^{-(\ell+d / 2)+\varepsilon}|x-y|^{l} \quad \forall \varepsilon>0 .
$$

При $\varepsilon<\ell$ получаем конечность интеграла (49) .

Остается доказать сходимость интеграла (50). Поскольку символ $p(x, \xi) \in$ $S_{\rho, \delta}^{1}$ эллиптичен, символ $b(x, \xi, h)$ принадлежит $S_{\rho, \delta}^{-(m+h)}$ и

$$
\left|\nabla_{x} b(x, \xi, h)\right| \leq C\langle\xi\rangle^{-(m+h)+\delta}
$$

Известное мультипликативное неравенство для гёльдеровских норм дает оценку

$$
\begin{aligned}
|b(x, \xi, h)-b(y, \xi, h)| \leq C \max _{x}\left|\nabla_{x} b(x, \xi, h)\right|^{l} \max _{x}|b(x, \xi, h)|^{1-l}|x-y|^{l} & \leq C\langle\xi\rangle^{-(m+h)+\delta l}|x-y|^{l} .
\end{aligned}
$$

Таким образом, интеграл (50) не превосходит

$$
C \int_{\substack{\mathbb{R}^{d} \times \Omega \times \Omega,|x-y|<1 / 2}} \frac{|\log | x-y||^{2 q}}{\langle\xi\rangle^{d+2(1-\delta) \ell}|x-y|^{d-2(l-\ell)}} d x d y d \xi<\infty .
$$

Лемма 5.2 и тем самым теорема 5.1 доказаны.

Закончим доказательство теоремы 1.3. В соответствии с замечанием 2 к лемме 2.1 оценка (39) эквивалентна оценке функции распределения $N\left(t, A_{h_{1}}\right)=$ $O\left(t^{d / m}(\log t)^{-q d / m}\right)$. Утверждение теоремы 1.3 следует из этой оценки и формул (36),(37) с помощью леммы 2.1.

Благодарность. Автор признателен А. И. Назарову за полезные обсуждения. 


\section{ЛИТЕРАТУРА}

1. Бирман М. Ш. Соломяк М. З. Асимптотика спектра псевдодифференциальных операторов с анизотропно-однородными символами // Вестн. ЛГУ. Сер. мат. 1977. № 13. С. 13-21.

2. Бирман М. Ш. Соломяк М. З. Асимптотика спектра псевдодифференциальных операторов с анизотропно-однородными символами. II // Вестн. ЛГУ. Сер. мат. 1979. № 13. C. $5-10$.

3. Dauge M., Robert D. Formule de Weyl pour une classe d'opérateurs pseudodifférentiels d'ordre négatif sur $L_{2}\left(\mathbb{R}^{n}\right) / /$ C. R. Acad. Sc. Paris Ser. I. 1986. V. 302, N 5. P. 175-178.

4. Dauge M., Robert D. Wey1's formula for a class of pseudo-differential operators with negative order in $L_{2}\left(\mathbb{R}^{n}\right) / /$ Lecture Notes Math. 1987. V. 1256. P. 91-122.

5. Karol' A. I. Asymptotic behavior of the spectrum of pseudodifferential operators of variable order // J. Math. Sci. 2015. V. 207, N 2. P. 236-248.

6. Karol' A. I. Asymptotics of spectra of compact pseudodifferential operators with nonsmooth symbols with respect to spatial variables // J. Math. Sci. 2017. V. 226, N 4. P. 355-374.

7. Benassi A., Jaffard S., Roux D. Gaussian processes and pseudodifferential elliptic operators // Rev. Mat. Iberoam. 1997. V. 13, N 1. P. 19-81.

8. Coeurjolly J.-F. Identification of multifractional Brownian motion // Bernoulli. 2005. V. 11, N 6. P. 987-1008.

9. Кароль А. И. Асимптотика сингулярных чисел компактных ПДО с символом, негладким по пространственным переменным // Функц. анализ и его прил. 2019. Т. 53, № 4. С. 89-92.

10. Lieberman G. Regularized distance and its applications // Pacific J. Math. 1985. V. 117, N 2. P. 329-352.

11. Бирман М. Ш. Соломяк М. З. Оценки сингулярных чисел интегральных операторов // Успехи мат. наук. 1977. Т. 32, № 1. С. 17-84.

12. Бирман М. Ш., Соломяк M. З. Спектральная теория самосопряженных операторов в гильбертовом пространстве. СПб: Лань, 2010.

13. Вайдль Т. Общие операторные идеалы слабого типа // Алгебра и анализ. 1992. Т. 4, № 3. C. $117-144$.

14. Берг Й., Лёфстрём Й. Интерполяционные пространства. Введение. М.: Мир, 1980.

15. Хёрмандер Л. Анализ линейных дифференциальных операторов с частными производными. М.: Мир, 1986. Т. 2.

Поступила в редакиию 28 октября 2019 г.

После доработки 24 марта 2020 г.

Принята к публикации 8 апреля 2020 г.

Кароль Андрей Игоревич

Санкт-Петербургский государственный университет,

Университетский пр., 28, Санкт-Петербург, Старый Петергоф 198504

andrey.i.karol@gmail.com 